\title{
REFERENCES
}

Chapman, G.B., \& Elstein, A.S. (2000). Cognitive processes and biases in medical decision making. In G.B. Chapman, \& F. Sonnenberg (Eds.). Decision making in health care: theory, psychology, and applications (pp. 183-210) New York: Cambridge University Press.

Elstein, A.S., \& Schwartz, A. (2000). Clinical reasoning in medicine. In J. Higgs \& M. Jones (Eds.). Clinical reasoning in the health professions (pp. 96-106) (2nd edn). Oxford: Butterworth-Heinemann.

Elstein, A.S., Shulman, L.S., \& Sprafka, S.A. (1978). Medical problem solving: An analysis of clinical reasoning. Cambridge, MA: Harvard University Press.

Hunink, M., Glasziou, P., Siegel, J., Weeks, J., Pliskin, J., Elstein, AS., Weinstein, MC. (in press). Decision making in health and medicine: Integrating evidence and values. New York: Cambridge Univ. Press.

Leape, L. (1994). Error in medicine. Journal of the American Medical Association, 272, 1851-1857.

Rouse, D.J., \& Owen, J. (1998). Decision analysis. Clinical Obstetrics and Gynecology, 41, 282-295.

Sackett, D.L., Richardson, W.S., Rosenberg, W., \& Haynes, R.B. (1997). Evidence-based medicine: How to practice and teach EBM. New York: Churchill Livingstone.

Author's biography:

Arthur Elstein has been teaching and doing research in medical judgment and decision making for over 30 years. His current research interests include evaluating the effects of instruction in evidence-based medicine, measuring patients' utilities, relations between quality-of-life scales and utility scales, and the effects of decision analysis recommendations on treatment choices for prostate cancer patients.

Author's address:

Arthur S. Elstein, PhD, Department of Medical Education (m/c 591), University of Illinois at Chicago, 808 S. Wood Street, Chicago, IL 60612-7309, USA. E-mail: aelstein@uic.edu

DOI: $10.1002 / \mathrm{bdm} .387$

\section{Decision Making in Real Life}

\author{
RICHARD GONZALEZ* \\ University of Michigan, USA
}

The paper by Lipshitz, Klein, Orasanu, and Salas (this issue; hereafter referred to as LKOS) reviews an impressive and influential line of research on naturalistic decision making (NDM). The features that define the NDM approach include the study of expert decision makers, a focus on the process that experts use to make decisions, a focus on if-then rules that experts use to connect a particular situation to a course of action, an emphasis on models that are domain- and context-specific, and prescription that is informed by the abilities of the decision maker.

An example of a decision model that emerges from the NDM perspective is the recognition-primed decision model (RPD), which was initially developed from interviews of expert firefighters but has since been applied to the study of experts in other domains such as design engineers and commercial aviation pilots. From interviews of experts in these domains, it appears that proficient decision makers generate an initial feasible course of action, mentally simulate the process and consequences of that action, and if the simulation suggests that the course of action will work as desired (without 'unintended consequences'), they act. As LKOS point out, what is interesting about these observations is that proficient decision makers generate, evaluate, and act. Interestingly, there does not appear to be a process of comparing different courses of action, as would be expected from the traditional decision making view.

LKOS attempted to explain the NDM perspective by contrasting it with other perspectives in the field of decision making. I understand that this is a strategy of rhetoric - to latch onto features that distinguish one's own approach in order to highlight differences with other work. It may be that the difference between the NDM approach and other approaches is not as great as the paper by LKOS suggests. As I read the LKOS paper, I found many of the classic themes of decision making embedded in the very ideas LKOS argued were distinct from the classic approaches. For example, the

*Correspondence to: Richard Gonzalez, Department of Psychology, University of Michigan, Ann Arbor, MI 48109, USA. E-mail: gonzo@umich.edu 
idea of evaluating a course of action as feasible (without regard to optimality) smacks of Simon's (1957) notion of satisficing, and satisficing was a key concept that fed into the development of the heuristics and biases approach. The notion of matching in a situation-action matching decision rule is analogous to the similarity component of the 'representativeness heuristic' of Kahneman and Tversky. Further, in the broad sense, does not the RPD model itself describe a type of heuristic used by experts?

I am troubled by the dual moves of NDM to (1) forgo the concept of optimality and (2) attempt to be prescriptive. To me this is like trying to walk up the down escalator. Can decisions be improved if there is not a sense of optimal performance? LKOS's notion of prescription is empirically based in the sense that prescriptions are derived from what experts do and from what they are capable of doing. It may be possible to improve the performance of experts beyond their current level. It seems limiting to set one's standard against existing performance rather than attempt to find new standards that are feasible given the constraints of both the domain and the limitations of the human expert. Maybe one prescription would be to help experts generate multiple courses of action (time permitting, of course). I think that a more general view of optimality may be needed. Simon's work, alluded to above, is one example, but another one is the notion that the maximization function is relatively flat near the optimum so that different 'courses of action' may be quite close to the optimum, and the small additional benefit provided by the optimum may not justify the large additional search costs. The weaker notion of optimality could also include the idea that in some prediction problems it is more critical to use the right cues (predictors) than to use the optimal set of weights to combine those cues (e.g. Dawes, 1979).

LKOS imply that formal models cannot provide information about process. I disagree. Indeed, there has been much recent progress in defining a formal axiomatic model of case-based decision making (e.g. Gilboa and Schmeidler, 1995). A formal approach can enhance observational research and can provide a deep understanding of the process models that emerge from such observation. A good formal model of decision making should illuminate the choice as well as the process used by the decision maker.

One avenue of research that seems promising is to study how experts become experts. What is the time course and what does the learning curve look like? What information do experts use? Is it possible to predict during the novice stage (e.g. during training) which individuals are more likely to become the experts that outshine the others? While the answers to these kinds of questions are interesting on their own, I think that these answers may be able to inform the process models used in NDM. This would place new and more demanding constraints on theory construction and testing - the NDM process models would not only have to account for how experts make decisions but also account for how the individual becomes an expert.

I am sympathetic to the underlying thrust of the NDM approach. I believe strongly that decision researchers need to pay more attention to domain- and context-bound decisions, they need to pay attention to experts and how expertise is acquired, and they need to pay attention to time pressure. Theories, models, and observations are forcing us to ask richer questions about decision making and these questions have perhaps gone beyond what some of the standard paradigms in behavioral decision are able to answer (e.g., it is not clear that the standard gamble will continue being a useful paradigm to study the rich set of psychological questions we are currently asking about decision making under risk, or that the 'almanac question' will continue being an appropriate paradigm in which to study the questions researchers are asking about the origins and realworld impact of overconfidence). However, I am not ready to abandon completely the lab study of novices performing abstract, context-free tasks without time pressure. I believe those studies do contribute to our understanding.

I see the NDM approach not as a replacement of what is currently being done but a new tool that decision researchers can (and should) add to their arsenal. The ideas and techniques that have been developed within the NDM framework provide a way to assess and test new research questions. Testing variables such as context and time pressure do more than merely examine boundary conditions - they provide new ways to test theory, they give a new source from which new theories can be derived, and they force researchers to consider the constraints the real world imposes. In much the same way that in physics there is room at the table for research that abstracts a phenomenon into a vacuum and also room for research that considers more context-bound variables such as friction and air resistance, there should be room at the table for multiple (and complementary) methodologies and paradigms in decision making research. It is in this sense that I think LKOS went too far in attempting to distinguish their approach from more traditional approaches. To me, the key issue is whether the NDM framework contributes new tools that decision researchers can use to answer their research questions. On this, I tend to agree with LKOS that NDM has made a contribution to the study of decision making.

\section{REFERENCES}

Dawes, R.M. (1979). The robust beauty of improper linear models in decision making. American Psychologist, 34, 571-582.

Gilboa, I., \& Schmeidler, D. (1995). Case-based decision theory. Quarterly Journal of Economics, 110, $605-639$. Simon, H.A. (1957). Models of man. New York: Wiley. 
Author's biography:

Richard Gonzalez received his $\mathrm{PhD}$ from Stanford University and is currently at the University of Michigan Psychology Department. His research interests include decision making, social psychology, and mathematical modelling.

Author's address:

Richard Gonzalez, Department of Psychology, University of Michigan, Ann Arbor, MI 48109, USA. E-mail: gonzo@umich.edu

DOI: $10.1002 / \mathrm{bdm} .388$

\title{
One Mode of Decision Making (Research)- No Less, No More
}

\author{
HELMUT JUNGERMANN* \\ Technical University Berlin, Germany
}

\begin{abstract}
The naturalistic decision-making (NDM) movement has brought interesting domains to the attention of decision researchers and in return has introduced theoretical ideas of decision research into these other domains, usually in applied areas. It has encouraged researchers and practitioners, who would not usually meet, to cooperate. It has developed new perspectives on the decision-making process and new methods for studying this process. And it has stimulated lively discussions about what decisions really are and how they are really made. These are important contributions to the field. However, since the target paper presents these contributions perfectly well, I shall discuss the facets of NDM research that I personally find most challenging and that I find most interesting to challenge myself. These are
\end{abstract}

- The tasks and situations that NDM researchers investigate

- The theoretical approach, in particular pattern-matching theories

- The methods and data favored in NDM studies, in particular the use of verbal data and

- The prescriptive implications and perspectives.

\section{TASKS AND SITUATIONS}

The most important contribution of NDM researchers is, in my view, that they have 'discovered' decision tasks, situations, and environments to which behavioral decision research never paid attention. These situations are characterized by features such as time pressure, complexity, dynamics, etc. The tasks are repetitive in nature, which implies that a person - for example a pilot, tank commander, firefighter, anesthesiologist, surgeon, etc. — can develop expertise in performing them, i.e. in making these decisions. Making a decision means selecting the adequate, appropriate, or right option, and not comparing options and selecting the best. This may require cognitive processes that are at least partially different from the type of decision making which is investigated in behavioral decision research.

NDM researchers have argued convincingly that these decisions are arrived at in a way that is different from the ones investigated by decision research over the past decades. To my knowledge, neither NDM nor BDT researchers have ever tried to apply BDT models to NDM tasks; the assumption that these tasks need new theories is based more on intuition than on evidence.

As interesting as these decision tasks, situations, and environments are, they are nevertheless no more representative of the 'real world' or ecologically valid or 'naturalistic' than the decisions studied by 'traditional' behavioral decision researchers (such as purchase decisions, managerial decisions, investment decisions, career decisions, medical decisions, etc.). I think it is not incidental that the tasks investigated in NDM research are few, and that they are very much the same; their features are characteristic of only a very small set of decisions. These decisions may be important ones, but they are very specific and in fact not very representative of human decision making. Even for pilots and surgeons (the examples so often used by NDM researchers), complex decisions under time pressure are really very much the exception to the rule. As long as the generalizability of theories, methods, and findings of NDM research to other domains has not been demonstrated, they will be confined to this small domain. The claim to substitute for BDT approaches is at present not justified.

*Correspondence to: Helmut Jungermann, Institut für Psychologie, Technische Universität, Berlin, Franklinstrasse 5-7 (FS-1), 10587 Berlin, Germany. E-mail: heju@gp.tu-berlin.de 